\title{
Self Adaptive Viscosity-Type Inertial Extragradient Algorithms for Solving Variational Inequalities with Applications
}

\section{Bing $\operatorname{Tan}^{a}$ and Xiaolong Qin ${ }^{b}$}

\author{
${ }^{a}$ Institute of Fundamental and Frontier Sciences, University of Electronic \\ Science and Technology of China \\ 611731 Chengdu, Sichuan, China \\ ${ }^{b}$ Department of Mathematics, Zhejiang Normal University \\ 321004 Jinhua, Zhejiang, China \\ E-mail(corresp.): qxlxajh@163.com \\ E-mail: bingtan72@gmail.com
}

Received November 3, 2020; revised October 31, 2021; accepted November 1, 2021

\begin{abstract}
In this paper, we introduce two new inertial extragradient algorithms with non-monotonic stepsizes for solving monotone and Lipschitz continuous variational inequality problems in real Hilbert spaces. Strong convergence theorems of the suggested iterative schemes are established without the prior knowledge of the Lipschitz constant of the mapping. Finally, some numerical examples are provided to illustrate the efficiency and advantages of the proposed algorithms and compare them with some related ones.
\end{abstract}

Keywords: variational inequality problem, optimal control problem, inertial subgradient extragradient method, inertial Tseng extragradient method, viscosity method.

AMS Subject Classification: 47H09; 47H10; 47J20; 47J25; 49J15.

\section{Introduction}

Our interest in this paper is to investigate self-adaptive fast iterative algorithms for solving variational inequality problems in real Hilbert spaces. Recall that the variational inequality problem (in short, VIP) is expressed as follows:

$$
\text { find } x^{*} \in \mathcal{C}, \quad \text { such that }\left\langle A x^{*}, x-x^{*}\right\rangle \geq 0, \quad \forall x \in \mathcal{C} \text {, }
$$

Copyright (C) 2022 The Author(s). Published by Vilnius Gediminas Technical University This is an Open Access article distributed under the terms of the Creative Commons Attribution License (http://creativecommons.org/licenses/by/4.0/), which permits unrestricted use, distribution, and reproduction in any medium, provided the original author and source are credited. 
where $A: \mathcal{H} \rightarrow \mathcal{H}$ is a nonlinear mapping, $\mathcal{C}$ is a nonempty convex closed set in a real Hilbert space $\mathcal{H}$ embed with the inner product $\langle\cdot, \cdot\rangle$ and the induced norm $\|\cdot\|$. We denote the set of all $\operatorname{such} x^{*}$ by $\operatorname{VI}(\mathcal{C}, A)$ in this paper. Let us recall some nonlinear mappings in functional analysis. For all $x, y \in \mathcal{H}$, a mapping $A: \mathcal{H} \rightarrow \mathcal{H}$ is said to be (i) $L$-Lipschitz continuous with $L>0$ iff $\|A x-A y\| \leq L\|x-y\|$; (ii) $\eta$-strongly monotone if there exists $\eta>0$ such that $\langle A x-A y, x-y\rangle \geq \eta\|x-y\|$; (iii) monotone if $\langle A x-A y, x-y\rangle \geq 0$.

The theory of variational inequalities has become a suitable model to deal with many practical problems in various areas, such as medical imaging, machine learning, economics, and optimal control; see, e.g., [4,6,13]. Recently, many work of literature on iterative methods for solving variational inequality problems have been proposed and studied, see, e.g., [3, 9, 12, 17, 24, 27, 29] and the references therein. Among these methods for solving variational inequalities, the projection-based methods and their variant forms play an important role. The simplest and oldest projection-type algorithm is the classical projected-gradient method, which performs only one projection on the feasible set. However, the convergence of the method requires a strong hypothesis: strong monotonicity or inverse strong monotonicity on mapping $A$. To avoid this strong assumption, Korpelevich [14] proposed the following extragradient method (EGM):

$$
\left\{\begin{array}{l}
y_{n}=P_{\mathcal{C}}\left(x_{n}-\vartheta A x_{n}\right), \\
x_{n+1}=P_{\mathcal{C}}\left(x_{n}-\vartheta A y_{n}\right), \quad \forall n \geq 1,
\end{array}\right.
$$

where $\vartheta \in(0,1 / L)$, the mapping $A$ is monotone and $L$-Lipschitz continuous and $P_{\mathcal{C}}$ is the metric projection onto $\mathcal{C}$. It is known that the sequence generated by $(\mathrm{EGM})$ converges weakly to a solution of $(\mathrm{VIP})$ provided that $\mathrm{VI}(\mathcal{C}, A) \neq \emptyset$. It is worth noting that the extragradient method needs to calculate two orthogonal projections on the feasible set in each iteration. This method is particularly useful if the feasible set is very simple so that projection can be performed easily. However, if the feasible set is a general closed and convex set, then the minimum distance problem must be solved twice to obtain the next iteration. This might increase the computational burden of the computer and further seriously affect the efficiency of the extragradient method. Next, we introduce two notable methods to overcome this difficulty. The first is the Tseng's extragradient method proposed by Tseng [31]:

$$
\left\{\begin{array}{l}
y_{n}=P_{\mathcal{C}}\left(x_{n}-\vartheta A x_{n}\right), \\
x_{n+1}=y_{n}-\vartheta\left(A y_{n}-A x_{n}\right), \quad \forall n \geq 1,
\end{array}\right.
$$

where $\vartheta \in(0,1 / L)$ and the mapping $A$ is monotone and $L$-Lipschitz continuous. Another well-known method is the subgradient extragradient method (SEGM) proposed by Censor, Gibali and Reich [5], which can be regarded as an improvement of the EGM. Their method is of the form:

$$
\left\{\begin{array}{l}
y_{n}=P_{\mathcal{C}}\left(x_{n}-\vartheta A x_{n}\right), \\
T_{n}=\left\{x \in \mathcal{H} \mid\left\langle x_{n}-\vartheta A x_{n}-y_{n}, x-y_{n}\right\rangle \leq 0\right\} \\
x_{n+1}=P_{T_{n}}\left(x_{n}-\vartheta A y_{n}\right), \quad \forall n \geq 1,
\end{array}\right.
$$


where $\vartheta \in(0,1 / L)$ and the mapping $A$ is monotone and $L$-Lipschitz continuous. They replaced the second projection onto the feasible set of EGM by a projection onto a specific constructible half-space. Note that both methods (TEGM) and (SEGM) have proven to obtain weak convergence in real Hilbert spaces. We know that strong convergence is preferable to weak convergence in infinitedimensional spaces. Recently, Kraikaew and Saejung [15] proposed a Halpern subgradient extragraduent method (HSEGM) to solve (VIP) in real Hilbert spaces. The method is inspired by the Halpern method and the (SEGM). Indeed, their method is of the form:

$$
\left\{\begin{array}{l}
y_{n}=P_{\mathcal{C}}\left(x_{n}-\vartheta A x_{n}\right), \\
T_{n}=\left\{x \in \mathcal{H} \mid\left\langle x_{n}-\vartheta A x_{n}-y_{n}, x-y_{n}\right\rangle \leq 0\right\}, \\
x_{n+1}=\varphi_{n} x_{1}+\left(1-\varphi_{n}\right) P_{T_{n}}\left(x_{n}-\vartheta A y_{n}\right), \quad \forall n \geq 1,
\end{array}\right.
$$

where $\vartheta \in(0,1 / L), \varphi_{n} \subset(0,1), \lim _{n \rightarrow \infty} \varphi_{n}=0, \sum_{n=1}^{\infty} \varphi_{n}=+\infty$ and the mapping $A$ is monotone and $L$-Lipschitz continuous. They proved that the iterative sequence $\left\{x_{n}\right\}$ defined by (1.1) converges to the unique solution of (VIP) in norm. Note that all the above methods require to know the Lipschitz constant of the mapping $A$. In 2017, Shehu and Iyiola [23] proposed a modification of SEGM with adoption of Armijo-like step size rule for solving (VIP), that is,

$$
\left\{\begin{array}{l}
y_{n}=P_{\mathcal{C}}\left(x_{n}-\vartheta_{n} A x_{n}\right), \\
T_{n}=\left\{x \in \mathcal{H} \mid\left\langle x_{n}-\vartheta_{n} A x_{n}-y_{n}, x-y_{n}\right\rangle \leq 0\right\}, \\
z_{n}=P_{T_{n}}\left(x_{n}-\vartheta_{n} A y_{n}\right), \\
x_{n+1}=\varphi_{n} f\left(x_{n}\right)+\left(1-\varphi_{n}\right) z_{n}, \quad \forall n \geq 1,
\end{array}\right.
$$

where the mapping $A$ is monotone and $L$-Lipschitz continuous, the mapping $f: \mathcal{H} \rightarrow \mathcal{H}$ is $k$-contraction, $\varphi_{n} \subset(0,1), \lim _{n \rightarrow \infty} \varphi_{n}=0, \sum_{n=1}^{\infty} \varphi_{n}=+\infty$, $\vartheta_{n}=\rho^{l_{n}}$ and $l_{n}$ is the smallest nonnegative inter such that $\vartheta_{n}\left\|A x_{n}-A y_{n}\right\| \leq$ $\mu\left\|x_{n}-y_{n}\right\|, \rho \in(0,1)$ and $\mu \in(0,1)$. Inspired by the work of [23], Thong and Hieu in their work [30] introduced a modification of Tseng's extragradient method, that is,

$$
\left\{\begin{array}{l}
y_{n}=P_{\mathcal{C}}\left(x_{n}-\vartheta_{n} x_{n}\right) \\
z_{n}=y_{n}-\vartheta_{n}\left(A y_{n}-A x_{n}\right) \\
x_{n+1}=\varphi_{n} f\left(x_{n}\right)+\left(1-\varphi_{n}\right) z_{n}, \quad \forall n \geq 1
\end{array}\right.
$$

where the mapping $A$ is monotone and $L$-Lipschitz continuous, the mapping $f: \mathcal{H} \rightarrow \mathcal{H}$ is $k$-contraction, $\varphi_{n} \subset(0,1), \lim _{n \rightarrow \infty} \varphi_{n}=0, \sum_{n=1}^{\infty} \varphi_{n}=+\infty$, $\vartheta_{n}$ is chosen to be the largest $\vartheta \in\left\{\gamma, \gamma l, \gamma l^{2}, \ldots\right\}$ satisfying $\vartheta\left\|A x_{n}-A y_{n}\right\| \leq$ $\mu\left\|x_{n}-y_{n}\right\|, \gamma>0, l \in(0,1)$ and $\mu \in(0,1)$. Both methods (1.2) and (1.3) can work without the prior knowledge of the Lipschitz constant of the mapping, but the Armijo-like step size may involve additional computation of the mapping A. Recently, Yang et al. [33,34] introduced two self-adaptive extragradient algorithms for solving variational inequalities. The algorithms are inspired by the subgradient extragradient method, the Tseng's extragradient method, the viscosity method and the new simple step size methods. Indeed, their 
algorithms generate two sequences $\left\{x_{n}\right\}$ by the following iterative schemes:

$$
\left\{\begin{array}{l}
y_{n}=P_{\mathcal{C}}\left(x_{n}-\vartheta_{n} A x_{n}\right), \\
T_{n}=\left\{x \in \mathcal{H} \mid\left\langle x_{n}-\vartheta_{n} A x_{n}-y_{n}, x-y_{n}\right\rangle \leq 0\right\}, \\
z_{n}=P_{T_{n}}\left(x_{n}-\vartheta_{n} A y_{n}\right), \\
x_{n+1}=\varphi_{n} x_{1}+\left(1-\varphi_{n}\right) z_{n}, \quad \forall n \geq 1,
\end{array}\right.
$$

where $\left\{\vartheta_{n}\right\}$ is updated by the following:

$$
\vartheta_{n+1}= \begin{cases}\min \left\{\frac{\mu\left(\left\|x_{n}-y_{n}\right\|^{2}+\left\|z_{n}-y_{n}\right\|^{2}\right)}{2\left\langle A x_{n}-A y_{n}, z_{n}-y_{n}\right\rangle}, \vartheta_{n}\right\}, & \text { if }\left\langle A x_{n}-A y_{n}, z_{n}-y_{n}\right\rangle>0 \\ \vartheta_{n}, & \text { otherwise, }\end{cases}
$$

and

$$
\left\{\begin{array}{l}
y_{n}=P_{\mathcal{C}}\left(x_{n}-\vartheta_{n} A x_{n}\right) \\
z_{n}=y_{n}-\vartheta_{n}\left(A y_{n}-A x_{n}\right), \\
x_{n+1}=\varphi_{n} f\left(x_{n}\right)+\left(1-\varphi_{n}\right) z_{n}, \quad \forall n \geq 1
\end{array}\right.
$$

where $\left\{\vartheta_{n}\right\}$ is updated by the following:

$$
\vartheta_{n+1}= \begin{cases}\min \left\{\frac{\mu\left\|x_{n}-y_{n}\right\|}{\left\|A x_{n}-A y_{n}\right\|}, \vartheta_{n}\right\}, & \text { if } A x_{n}-A y_{n} \neq 0, \\ \vartheta_{n}, & \text { otherwise. }\end{cases}
$$

In Algorithms (1.4) and (1.5), the mapping $A$ is $L$-Lipschitz continuous and monotone, the mapping $f: \mathcal{H} \rightarrow \mathcal{H}$ is $k$-contraction, $\varphi_{n} \subset(0,1), \lim _{n \rightarrow \infty} \varphi_{n}=$ $0, \sum_{n=1}^{\infty} \varphi_{n}=+\infty, \vartheta_{1} \in(0,1)$ and $\mu \in(0,1)$. It should be noted that Algorithms (1.2)-(1.5) have established strong convergence theorems in real Hilbert spaces. Moreover, the stepsizes used in Algorithms (1.4) and (1.5) are non-increasing, which may affect the execution efficiency of such algorithms.

On the other hand, in recent years, there has been tremendous interest in developing fast iterative algorithms. Many authors have used inertial technology to build a large number of iterative algorithms that can improve the convergence speed; see, e.g., $[2,7,10,11,21,22,25]$ and the references therein. One of the common features of these algorithms is that the next iteration depends on the combination of the previous two iterations. Note that this minor change greatly improves the performance of the algorithms.

Motivated and inspired by the above work, in this paper, we present two inertial extragradient algorithms with non-monotonic stepsizes for solving the monotone variational inequality problem in real Hilbert spaces. Our algorithms do not require the prior knowledge of the Lipschitz constant of the mapping. Strong convergence theorems of our algorithms are established under some suitable conditions. Finally, we provide some numerical experiments to support the theoretical results. The two algorithms obtained in this paper improve and extend some related results in this field [15,23,30,33,34].

The structure of the paper is as follows. In Section 2, we present some preliminaries that will be needed in the sequel. In Section 3, we propose two algorithms and analyze their convergence. In Section 4, some numerical examples are provided to illustrate the numerical behavior of the proposed algorithms. Finally, we conclude this paper with a brief summary in Section 5, the last section. 


\section{Preliminaries}

Let $\mathcal{C}$ be a nonempty closed and convex subset of a real Hilbert space $\mathcal{H}$. The weak convergence and strong convergence of $\left\{x_{n}\right\}_{n=1}^{\infty}$ to $x$ are represented by $x_{n} \rightarrow x$ and $x_{n} \rightarrow x$, respectively. For each $x, y \in \mathcal{H}$ and $\delta \in \mathbb{R}$, we have the following facts:

1. $\|x+y\|^{2}=\|x\|^{2}+2\langle x, y\rangle+\|y\|^{2}$;

2. $\|x+y\|^{2} \leq\|x\|^{2}+2\langle y, x+y\rangle$;

3. $\|\delta x+(1-\delta) y\|^{2}=\delta\|x\|^{2}+(1-\delta)\|y\|^{2}-\delta(1-\delta)\|x-y\|^{2}$.

For every point $x \in \mathcal{H}$, there exists a unique nearest point in $\mathcal{C}$, denoted by $P_{\mathcal{C}}(x)$ such that $P_{\mathcal{C}}(x):=\operatorname{argmin}\{\|x-y\|, y \in \mathcal{C}\} . P_{\mathcal{C}}$ is called the metric projection of $\mathcal{H}$ onto $\mathcal{C}$. It is known that $P_{\mathcal{C}}$ has the following basic properties:

- $\left\langle x-P_{\mathcal{C}}(x), y-P_{\mathcal{C}}(x)\right\rangle \leq 0, \forall y \in \mathcal{C}$;

- $\left\|P_{\mathcal{C}}(x)-P_{\mathcal{C}}(y)\right\|^{2} \leq\left\langle P_{\mathcal{C}}(x)-P_{\mathcal{C}}(y), x-y\right\rangle, \forall y \in \mathcal{H}$.

We state the following well-known lemmas which will be used in the sequel.

Lemma 1. ( [26]) Let $\mathcal{C}$ be a nonempty, convex and closed subset of a real Hilbert space $\mathcal{H}$ and $A: \mathcal{C} \rightarrow \mathcal{H}$ be a continuous and monotone mapping. Then, $x^{*}$ is a solution of $\operatorname{VI}(\mathcal{C}, A)$ if and only if $\left\langle A x, x-x^{*}\right\rangle \geq 0, \forall x \in \mathcal{C}$.

Lemma 2. ( $[15])$ Let $A: \mathcal{H} \rightarrow \mathcal{H}$ be a monotone and L-Lipschitz continuous mapping on $\mathcal{C}$. Let $S=P_{\mathcal{C}}(I-\mu A)$, where $\mu>0$. If $\left\{x_{n}\right\}$ is a sequence in $\mathcal{H}$ satisfying $x_{n} \rightarrow q$ and $x_{n}-S x_{n} \rightarrow 0$, then $q \in \operatorname{VI}(\mathcal{C}, A)=\operatorname{Fix}(S)$.

Lemma 3. ( [20]) Let $\left\{a_{n}\right\}$ be a sequence of nonnegative real numbers, $\left\{\tau_{n}\right\}$ be a sequence of real numbers in $(0,1)$ with $\sum_{n=1}^{\infty} \tau_{n}=\infty$, and $\left\{b_{n}\right\}$ be a sequence of real numbers. Assume that $a_{n+1} \leq b_{n} \tau_{n}+\left(1-\tau_{n}\right) a_{n}, \forall n \geq 1$. If $\limsup _{k \rightarrow \infty} b_{n_{k}} \leq 0$ for every subsequence $\left\{a_{n_{k}}\right\}$ of $\left\{a_{n}\right\}$ satisfying $\lim \inf _{k \rightarrow \infty}$ $\left(a_{n_{k}+1}-a_{n_{k}}\right) \geq 0$, then $\lim _{n \rightarrow \infty} a_{n}=0$.

\section{Main results}

In this section, we propose two new inertial algorithms for monotone variational inequalities, which are based on the subgradient extragradient method, the Tseng's extragradient method and the viscosity method. First, we assume that our algorithms satisfy the following conditions.

(C1) The feasible set $\mathcal{C}$ is a nonempty, closed and convex subset of $\mathcal{H}$.

(C2) The solution set of the (VIP) is nonempty, that is $\operatorname{VI}(\mathcal{C}, A) \neq \emptyset$.

(C3) The mapping $A: \mathcal{H} \rightarrow \mathcal{H}$ is monotone and $L$-Lipschitz continuous on $\mathcal{H}$.

(C4) The mapping $f: \mathcal{H} \rightarrow \mathcal{H}$ is $k$-contraction with constant $k \in[0,1)$.

(C5) Let $\left\{\epsilon_{n}\right\}$ be a positive sequence such that $\lim _{n \rightarrow \infty} \frac{\epsilon_{n}}{\varphi_{n}}=0$, where sequence $\left\{\varphi_{n}\right\} \subset(0,1)$ satisfies $\lim _{n \rightarrow \infty} \varphi_{n}=0$ and $\sum_{n=1}^{\infty} \varphi_{n}=\infty$. 


\subsection{The self adaptive viscosity-type inertial subgradient extragra- dient algorithm}

Now, we introduce a self adaptive viscosity-type inertial subgradient extragradient algorithm to solve (VIP). The Algorithm 3.1 is described as follows.

Algorithm 3.1. Viscosity-type inertial subgradient extragradient algorithm

Initialization: Set $\varrho>0, \vartheta_{1}>0, \mu \in(0,1)$. Choose a nonnegative real sequence $\left\{\xi_{n}\right\}$ such that $\sum_{n=1}^{\infty} \xi_{n}<+\infty$. Let $x_{0}, x_{1} \in \mathcal{H}$ be arbitrarily.

Iterative Steps: Calculate $x_{n+1}$ as follows:

$$
\left\{\begin{array}{l}
u_{n}=x_{n}+\varrho_{n}\left(x_{n}-x_{n-1}\right), \\
y_{n}=P_{\mathcal{C}}\left(u_{n}-\vartheta_{n} A u_{n}\right), \\
T_{n}=\left\{z \in \mathcal{H}:\left\langle u_{n}-\vartheta_{n} A u_{n}-y_{n}, z-y_{n}\right\rangle \leq 0\right\} \\
z_{n}=P_{T_{n}}\left(u_{n}-\vartheta_{n} A y_{n}\right), \\
x_{n+1}=\varphi_{n} f\left(x_{n}\right)+\left(1-\varphi_{n}\right) z_{n}, \quad \forall n \geq 1,
\end{array}\right.
$$

where $\left\{\varrho_{n}\right\}$ and $\left\{\vartheta_{n}\right\}$ are updated by (3.1) and (3.2), respectively.

$$
\begin{aligned}
\varrho_{n} & = \begin{cases}\min \left\{\frac{\epsilon_{n}}{\left\|x_{n}-x_{n-1}\right\|}, \varrho\right\}, & \text { if } x_{n} \neq x_{n-1} ; \\
\varrho, & \text { otherwise. }\end{cases} \\
\vartheta_{n+1} & = \begin{cases}\min \left\{\frac{\mu\left\|u_{n}-y_{n}\right\|}{\left\|A u_{n}-A y_{n}\right\|}, \vartheta_{n}+\xi_{n}\right\}, & \text { if } A u_{n}-A y_{n} \neq 0 ; \\
\vartheta_{n}+\xi_{n}, & \text { otherwise. }\end{cases}
\end{aligned}
$$

Remark 1. It follows from (3.1) that

$$
\lim _{n \rightarrow \infty} \frac{\varrho_{n}}{\varphi_{n}}\left\|x_{n}-x_{n-1}\right\|=0 .
$$

Indeed, we have $\varrho_{n}\left\|x_{n}-x_{n-1}\right\| \leq \epsilon_{n}$ for all $n$, which together with $\lim _{n \rightarrow \infty} \frac{\epsilon_{n}}{\varphi_{n}}=$ 0 implies that

$$
\lim _{n \rightarrow \infty} \frac{\varrho_{n}}{\varphi_{n}}\left\|x_{n}-x_{n-1}\right\| \leq \lim _{n \rightarrow \infty} \frac{\epsilon_{n}}{\varphi_{n}}=0 .
$$

The following lemmas are quite helpful to analyze the convergence of the algorithm.

Lemma 4. Suppose that Assumptions (C1)-(C3) hold. The sequence $\left\{\vartheta_{n}\right\}$ generated by $(3.2)$ is well defined and $\lim _{n \rightarrow \infty} \vartheta_{n}=\vartheta$ and $\vartheta \in\left[\min \left\{\frac{\mu}{L}, \vartheta_{1}\right\}, \vartheta_{1}+\Xi\right]$, where $\Xi=\sum_{n=1}^{\infty} \xi_{n}$.

Proof. Since mapping $M$ is $L$-Lipschitz continuous, one has,

$$
\frac{\mu\left\|u_{n}-y_{n}\right\|}{\left\|A u_{n}-A y_{n}\right\|} \geq \frac{\mu\left\|u_{n}-y_{n}\right\|}{L\left\|u_{n}-y_{n}\right\|}=\frac{\mu}{L}, \text { if } A u_{n} \neq A y_{n}
$$

Thus, $\vartheta_{n} \geq \min \left\{\frac{\mu}{L}, \vartheta_{1}\right\}$. It follows from the definition of $\vartheta_{n+1}$ that $\vartheta_{n+1} \leq$ $\vartheta_{1}+\Xi$. Consequently, the sequence $\left\{\vartheta_{n}\right\}$ defined in (3.2) is bounded 
and $\vartheta_{n} \in\left[\min \left\{\frac{\mu}{L}, \vartheta_{1}\right\}, \vartheta_{1}+\Xi\right]$. For simplicity, we define $\left(\vartheta_{n+1}-\vartheta_{n}\right)^{+}=$ $\max \left\{0, \vartheta_{n+1}-\vartheta_{n}\right\}$ and $\left(\vartheta_{n+1}-\vartheta_{n}\right)^{-}=\max \left\{0,-\left(\vartheta_{n+1}-\vartheta_{n}\right)\right\}$. By the definition of $\left\{\vartheta_{n}\right\}$, one obtains $\sum_{n=1}^{\infty}\left(\vartheta_{n+1}-\vartheta_{n}\right)^{+} \leq \sum_{n=1}^{\infty} \xi_{n}<+\infty$, which implies that the series $\sum_{n=1}^{\infty}\left(\vartheta_{n+1}-\vartheta_{n}\right)^{+}$is convergent. Next we show the convergence of the series $\sum_{n=1}^{\infty}\left(\vartheta_{n+1}-\vartheta_{n}\right)^{-}$. Suppose that $\sum_{n=1}^{\infty}\left(\vartheta_{n+1}-\vartheta_{n}\right)^{-}=$ $+\infty$. Note that $\vartheta_{n+1}-\vartheta_{n}=\left(\vartheta_{n+1}-\vartheta_{n}\right)^{+}-\left(\vartheta_{n+1}-\vartheta_{n}\right)^{-}$. Therefore,

$$
\vartheta_{k+1}-\vartheta_{1}=\sum_{n=1}^{k}\left(\vartheta_{n+1}-\vartheta_{n}\right)=\sum_{n=1}^{k}\left(\vartheta_{n+1}-\vartheta_{n}\right)^{+}-\sum_{n=1}^{k}\left(\vartheta_{n+1}-\vartheta_{n}\right)^{-} .
$$

Taking $k \rightarrow+\infty$ in the above equation, we get $\lim _{k \rightarrow+\infty} \vartheta_{k} \rightarrow-\infty$. That is a contradiction. Hence, we obtain $\lim _{n \rightarrow \infty} \vartheta_{n}=\vartheta, \vartheta \in\left[\min \left\{\frac{\mu}{L}, \vartheta_{1}\right\}, \vartheta_{1}+\Xi\right]$.

Remark 2. The idea of the step size $\vartheta_{n}$ defined in (3.2) is derived from [16]. It is worth noting that the step size $\vartheta_{n}$ generated in Algorithm 3.1 is allowed to increase when the iteration increases. Therefore, the use of this type of step size reduces the dependence on the initial step size $\vartheta_{1}$. On the other hand, because of $\sum_{n=1}^{\infty} \xi_{n}<+\infty$, which implies that $\lim _{n \rightarrow \infty} \xi_{n}=0$. Consequently, the step size $\vartheta_{n}$ may not increase when $n$ is large enough. If $\xi_{n}=0$, then the step size $\vartheta_{n}$ in Algorithm 3.1 is similar to the approaches in $[1,2,33]$.

The following Lemma 5 plays an important role in the convergence analysis of the Algorithm 3.1 and it can be easily obtained by using the same statement as Lemma 3.2 in [28].

Lemma 5. ( [28]) Assume that Conditions (C1)-(C3) hold. Let $\left\{z_{n}\right\}$ be a sequence generated by Algorithm 3.1. Then

$$
\left\|z_{n}-p\right\|^{2} \leq\left\|u_{n}-p\right\|^{2}-\left(1-\mu \frac{\vartheta_{n}}{\vartheta_{n+1}}\right)\left(\left\|y_{n}-u_{n}\right\|^{2}+\left\|z_{n}-y_{n}\right\|^{2}\right)
$$

for all $p \in \operatorname{VI}(\mathcal{C}, A)$.

Theorem 1. Assume that Conditions (C1)-(C5) hold. Then the sequence $\left\{x_{n}\right\}$ created by Algorithm 3.1 converges to an element $p \in \operatorname{VI}(\mathcal{C}, A)$ in norm, where $p=P_{\mathrm{VI}(\mathcal{C}, A)}(f(p))$.

Proof. According to Lemma 4, it follows that

$$
\lim _{n \rightarrow \infty}\left(1-\mu \vartheta_{n} / \vartheta_{n+1}\right)=1-\mu>0
$$

Hence, there exists $n_{0} \in \mathbb{N}$, such that

$$
1-\mu \vartheta_{n} / \vartheta_{n+1}>0, \quad \forall n \geq n_{0} .
$$

Combining Lemma 5 and (3.3), we obtain

$$
\left\|z_{n}-p\right\| \leq\left\|u_{n}-p\right\|, \quad \forall n \geq n_{0} .
$$


Claim 1. The sequence $\left\{x_{n}\right\}$ is bounded. Indeed, from the definition of $u_{n}$, we have

$$
\left\|u_{n}-p\right\| \leq\left\|x_{n}-p\right\|+\varphi_{n}\left(\varrho_{n} / \varphi_{n}\right)\left\|x_{n}-x_{n-1}\right\| .
$$

By Remark 1, we have $\frac{\varrho_{n}}{\varphi_{n}}\left\|x_{n}-x_{n-1}\right\| \rightarrow 0$ as $n \rightarrow \infty$. Thus, there exists a constant $M_{1}>0$ such that

$$
\frac{\varrho_{n}}{\varphi_{n}}\left\|x_{n}-x_{n-1}\right\| \leq M_{1}, \quad \forall n \geq 1 .
$$

From (3.4), (3.5) and (3.6), we find that

$$
\left\|z_{n}-p\right\| \leq\left\|u_{n}-p\right\| \leq\left\|x_{n}-p\right\|+\varphi_{n} M_{1}, \quad \forall n \geq n_{0} .
$$

Using the definition of $x_{n+1}$ and (3.7), we get

$$
\begin{aligned}
\left\|x_{n+1}-p\right\| & =\left\|\varphi_{n}\left(f\left(x_{n}\right)-p\right)+\left(1-\varphi_{n}\right)\left(z_{n}-p\right)\right\| \\
& \leq \varphi_{n}\left\|f\left(x_{n}\right)-f(p)\right\|+\varphi_{n}\|f(p)-p\|+\left(1-\varphi_{n}\right)\left\|z_{n}-p\right\| \\
& \leq \varphi_{n} \kappa\left\|x_{n}-p\right\|+\varphi_{n}\|f(p)-p\|+\left(1-\varphi_{n}\right)\left\|z_{n}-p\right\| \\
& \leq\left(1-(1-\kappa) \varphi_{n}\right)\left\|x_{n}-p\right\|+(1-\kappa) \varphi_{n} \frac{M_{1}+\|f(p)-p\|}{1-\kappa} \\
& \leq \max \left\{\left\|x_{n}-p\right\|, \frac{M_{1}+\|f(p)-p\|}{1-\kappa}\right\} \\
& \leq \cdots \leq \max \left\{\left\|x_{n_{0}}-p\right\|, \frac{M_{1}+\|f(p)-p\|}{1-\kappa}\right\} .
\end{aligned}
$$

This implies that $\left\{x_{n}\right\}$ is bounded. So $\left\{u_{n}\right\},\left\{z_{n}\right\}$ and $\left\{f\left(x_{n}\right)\right\}$ are also bounded.

\section{Claim 2.}

$$
\begin{aligned}
& \left(1-\varphi_{n}\right)\left[\left(1-\mu \frac{\vartheta_{n}}{\vartheta_{n+1}}\right)\left\|y_{n}-u_{n}\right\|^{2}+\left(1-\mu \frac{\vartheta_{n}}{\vartheta_{n+1}}\right)\left\|z_{n}-y_{n}\right\|^{2}\right] \\
& \leq\left\|x_{n}-p\right\|^{2}-\left\|x_{n+1}-p\right\|^{2}+\varphi_{n} M_{4}
\end{aligned}
$$

for some $M_{4}>0$. Indeed, in view of (3.7), one sees that

$$
\begin{aligned}
& \left\|u_{n}-p\right\|^{2} \leq\left(\left\|x_{n}-p\right\|+\varphi_{n} M_{1}\right)^{2} \\
& \quad=\left\|x_{n}-p\right\|^{2}+\varphi_{n}\left(2 M_{1}\left\|x_{n}-p\right\|+\varphi_{n} M_{1}^{2}\right) \leq\left\|x_{n}-p\right\|^{2}+\varphi_{n} M_{2}
\end{aligned}
$$

for some $M_{2}>0$. Combining Lemma 5 and (3.8), we obtain

$$
\begin{aligned}
\left\|x_{n+1}-p\right\|^{2} \leq & \varphi_{n}\left\|f\left(x_{n}\right)-p\right\|^{2}+\left(1-\varphi_{n}\right)\left\|z_{n}-p\right\|^{2} \\
\leq & \varphi_{n}\left(\left\|f\left(x_{n}\right)-f(p)\right\|+\|f(p)-p\|\right)^{2}+\left(1-\varphi_{n}\right)\left\|z_{n}-p\right\|^{2} \\
\leq & \varphi_{n}\left(\left\|x_{n}-p\right\|+\|f(p)-p\|\right)^{2}+\left(1-\varphi_{n}\right)\left\|z_{n}-p\right\|^{2} \\
= & \varphi_{n}\left\|x_{n}-p\right\|^{2}+\left(1-\varphi_{n}\right)\left\|z_{n}-p\right\|^{2} \\
& +\varphi_{n}\left(2\left\|x_{n}-p\right\| \cdot\|f(p)-p\|+\|f(p)-p\|^{2}\right) \\
\leq & \varphi_{n}\left\|x_{n}-p\right\|^{2}+\left(1-\varphi_{n}\right)\left\|z_{n}-p\right\|^{2}+\varphi_{n} M_{3} \\
\leq & \left\|x_{n}-p\right\|^{2}-\left(1-\varphi_{n}\right)\left[\left(1-\mu \vartheta_{n} / \vartheta_{n+1}\right)\left\|y_{n}-u_{n}\right\|^{2}\right. \\
& \left.+\left(1-\mu \vartheta_{n} / \vartheta_{n+1}\right)\left\|z_{n}-y_{n}\right\|^{2}\right]+\varphi_{n} M_{4},
\end{aligned}
$$


where $M_{4}:=M_{2}+M_{3}$. We can get the desired result through a simple conversion.

\section{Claim 3.}

$$
\begin{aligned}
\left\|x_{n+1}-p\right\|^{2} \leq & \left(1-(1-\kappa) \varphi_{n}\right)\left\|x_{n}-p\right\|^{2}+(1-\kappa) \varphi_{n} \cdot\left[\frac{3 M}{1-\kappa} \cdot \frac{\varrho_{n}}{\varphi_{n}}\left\|x_{n}-x_{n-1}\right\|\right. \\
& \left.+\frac{2}{1-\kappa}\left\langle f(p)-p, x_{n+1}-p\right\rangle\right], \quad \forall n \geq n_{0}
\end{aligned}
$$

for some $M>0$. Indeed, we have

$$
\begin{aligned}
\left\|u_{n}-p\right\|^{2} & =\left\|x_{n}+\varrho_{n}\left(x_{n}-x_{n-1}\right)-p\right\|^{2} \\
& \leq\left\|x_{n}-p\right\|^{2}+2 \varrho_{n}\left\|x_{n}-p\right\|\left\|x_{n}-x_{n-1}\right\|+\varrho_{n}^{2}\left\|x_{n}-x_{n-1}\right\|^{2} \\
& \leq\left\|x_{n}-p\right\|^{2}+3 M \varrho_{n}\left\|x_{n}-x_{n-1}\right\|,
\end{aligned}
$$

where $M:=\sup _{n \in \mathbb{N}}\left\{\left\|x_{n}-p\right\|, \varrho\left\|x_{n}-x_{n-1}\right\|\right\}>0$. Using (3.4) and (3.9), we obtain

$$
\begin{aligned}
& \left\|x_{n+1}-p\right\|^{2}=\left\|\varphi_{n}\left(f\left(x_{n}\right)-f(p)\right)+\left(1-\varphi_{n}\right)\left(z_{n}-p\right)+\varphi_{n}(f(p)-p)\right\|^{2} \\
& \leq\left\|\varphi_{n}\left(f\left(x_{n}\right)-f(p)\right)+\left(1-\varphi_{n}\right)\left(z_{n}-p\right)\right\|^{2}+2 \varphi_{n}\left\langle f(p)-p, x_{n+1}-p\right\rangle \\
& \leq \varphi_{n}\left\|f\left(x_{n}\right)-f(p)\right\|^{2}+\left(1-\varphi_{n}\right)\left\|z_{n}-p\right\|^{2}+2 \varphi_{n}\left\langle f(p)-p, x_{n+1}-p\right\rangle \\
& \leq \varphi_{n} \kappa\left\|x_{n}-p\right\|^{2}+\left(1-\varphi_{n}\right)\left\|u_{n}-p\right\|^{2}+2 \varphi_{n}\left\langle f(p)-p, x_{n+1}-p\right\rangle \\
& \leq\left(1-(1-\kappa) \varphi_{n}\right)\left\|x_{n}-p\right\|^{2}+(1-\kappa) \varphi_{n} \cdot\left[\frac{3 M}{1-\kappa} \cdot \frac{\varrho_{n}}{\varphi_{n}}\left\|x_{n}-x_{n-1}\right\|\right. \\
& \left.\quad+\frac{2}{1-\kappa}\left\langle f(p)-p, x_{n+1}-p\right\rangle\right], \quad \forall n \geq n_{0} .
\end{aligned}
$$

Claim 4. $\left\{\left\|x_{n}-p\right\|^{2}\right\}$ converges to zero. Indeed, from Lemma 3 and Remark 1, it suffices to show that $\limsup _{k \rightarrow \infty}\left\langle f(p)-p, x_{n_{k}+1}-p\right\rangle \leq 0$ for every subsequence $\left\{\left\|x_{n_{k}}-p\right\|\right\}$ of $\left\{\left\|x_{n}-p\right\|\right\}$ satisfying

$$
\liminf _{k \rightarrow \infty}\left(\left\|x_{n_{k}+1}-p\right\|-\left\|x_{n_{k}}-p\right\|\right) \geq 0
$$

For this purpose, we assume that $\left\{\left\|x_{n_{k}}-p\right\|\right\}$ is a subsequence of $\left\{\left\|x_{n}-p\right\|\right\}$ such that $\liminf \inf _{k \rightarrow \infty}\left(\left\|x_{n_{k}+1}-p\right\|-\left\|x_{n_{k}}-p\right\|\right) \geq 0$. Then

$$
\begin{aligned}
& \liminf _{k \rightarrow \infty}\left(\left\|x_{n_{k}+1}-p\right\|^{2}-\left\|x_{n_{k}}-p\right\|^{2}\right) \\
& \quad=\liminf _{k \rightarrow \infty}\left[\left(\left\|x_{n_{k}+1}-p\right\|-\left\|x_{n_{k}}-p\right\|\right)\left(\left\|x_{n_{k}+1}-p\right\|+\left\|x_{n_{k}}-p\right\|\right)\right] \geq 0 .
\end{aligned}
$$

It follows from Claim 2 and Condition (C5) that

$$
\begin{aligned}
& \limsup _{k \rightarrow \infty}\left\{( 1 - \varphi _ { n _ { k } } ) \left[\left(1-\mu \frac{\vartheta_{n_{k}}}{\vartheta_{n_{k}+1}}\right)\left\|y_{n_{k}}-u_{n_{k}}\right\|^{2}+\left(1-\mu \frac{\vartheta_{n_{k}}}{\vartheta_{n_{k}+1}}\right)\right.\right. \\
& \left.\left.\quad \times\left\|z_{n_{k}}-y_{n_{k}}\right\|^{2}\right]\right\} \leq \limsup _{k \rightarrow \infty}\left[\left\|x_{n_{k}}-p\right\|^{2}-\left\|x_{n_{k}+1}-p\right\|^{2}+\varphi_{n_{k}} M_{4}\right] \\
& \quad \leq \limsup _{k \rightarrow \infty}\left[\left\|x_{n_{k}}-p\right\|^{2}-\left\|x_{n_{k}+1}-p\right\|^{2}\right]+\limsup _{k \rightarrow \infty} \varphi_{n_{k}} M_{4} \\
& \quad=-\liminf _{k \rightarrow \infty}\left[\left\|x_{n_{k}+1}-p\right\|^{2}-\left\|x_{n_{k}}-p\right\|^{2}\right] \leq 0,
\end{aligned}
$$


which implies that

$$
\lim _{k \rightarrow \infty}\left\|y_{n_{k}}-u_{n_{k}}\right\|=0 \text { and } \lim _{k \rightarrow \infty}\left\|z_{n_{k}}-y_{n_{k}}\right\|=0 .
$$

Therefore, we obtain

$$
\lim _{k \rightarrow \infty}\left\|z_{n_{k}}-u_{n_{k}}\right\| \leq \lim _{k \rightarrow \infty}\left\|z_{n_{k}}-y_{n_{k}}\right\|+\lim _{k \rightarrow \infty}\left\|y_{n_{k}}-u_{n_{k}}\right\|=0 .
$$

Moreover, using Remark 1 and Condition (C5), we have

$$
\begin{aligned}
& \left\|x_{n_{k}}-u_{n_{k}}\right\|=\varrho_{n_{k}}\left\|x_{n_{k}}-x_{n_{k}-1}\right\|=\varphi_{n_{k}} \cdot \frac{\varrho_{n_{k}}}{\varphi_{n_{k}}}\left\|x_{n_{k}}-x_{n_{k}-1}\right\| \rightarrow 0, \\
& \left\|x_{n_{k}+1}-z_{n_{k}}\right\|=\varphi_{n_{k}}\left\|z_{n_{k}}-f\left(x_{n_{k}}\right)\right\| \rightarrow 0
\end{aligned}
$$

Thus, we conclude that

$$
\left\|x_{n_{k}+1}-x_{n_{k}}\right\| \leq\left\|x_{n_{k}+1}-z_{n_{k}}\right\|+\left\|z_{n_{k}}-u_{n_{k}}\right\|+\left\|u_{n_{k}}-x_{n_{k}}\right\| \rightarrow 0 .
$$

Since the sequence $\left\{x_{n_{k}}\right\}$ is bounded, there exists a subsequence $\left\{x_{n_{k_{j}}}\right\}$ of $\left\{x_{n_{k}}\right\}$ such that $x_{n_{k_{j}}} \rightarrow z$. Furthermore,

$$
\limsup _{k \rightarrow \infty}\left\langle f(p)-p, x_{n_{k}}-p\right\rangle=\lim _{j \rightarrow \infty}\left\langle f(p)-p, x_{n_{k_{j}}}-p\right\rangle=\langle f(p)-p, z-p\rangle .
$$

We get $u_{n_{k}} \rightarrow z$ since $\left\|x_{n_{k}}-u_{n_{k}}\right\| \rightarrow 0$. This together with $\lim _{k \rightarrow \infty} \| u_{n_{k}}-$ $y_{n_{k}} \|=0$ and Lemma 2, yields $z \in \operatorname{VI}(\mathcal{C}, A)$. From the definition of $p$ and (3.12), one sees that

$$
\limsup _{k \rightarrow \infty}\left\langle f(p)-p, x_{n_{k}}-p\right\rangle=\langle f(p)-p, z-p\rangle \leq 0 .
$$

Combining (3.11) and (3.13), we can show that

$$
\limsup _{k \rightarrow \infty}\left\langle f(p)-p, x_{n_{k}+1}-p\right\rangle \leq \limsup _{k \rightarrow \infty}\left\langle f(p)-p, x_{n_{k}}-p\right\rangle \leq 0 .
$$

Thus, from Remark 1, (3.14), Claim 3 and Lemma 3, we conclude that $x_{n} \rightarrow p$ as $n \rightarrow \infty$. That is the desired result.

\subsection{The self adaptive viscosity-type inertial Tseng extragradient algorithm}

In this subsection, we introduce a self adaptive viscosity-type inertial Tseng extragradient algorithm for solving (VIP). The Algorithm 3.2 is stated as follows.

Algorithm 3.2. Viscosity-type inertial Tseng extragradient algorithm

Initialization: Set $\varrho>0, \vartheta_{1}>0, \mu \in(0,1)$. Choose a nonnegative real sequence $\left\{\xi_{n}\right\}$ such that $\sum_{n=1}^{\infty} \xi_{n}<+\infty$. Let $x_{0}, x_{1} \in \mathcal{H}$ be arbitrarily.

Iterative Steps: Calculate $x_{n+1}$ as follows:

$$
\left\{\begin{array}{l}
u_{n}=x_{n}+\varrho_{n}\left(x_{n}-x_{n-1}\right), \\
y_{n}=P_{\mathcal{C}}\left(u_{n}-\vartheta_{n} A u_{n}\right), \\
z_{n}=y_{n}-\vartheta_{n}\left(A y_{n}-A u_{n}\right), \\
x_{n+1}=\varphi_{n} f\left(x_{n}\right)+\left(1-\varphi_{n}\right) z_{n}, \quad \forall n \geq 1,
\end{array}\right.
$$

where $\left\{\varrho_{n}\right\}$ and $\left\{\vartheta_{n}\right\}$ are updated by (3.1) and (3.2), respectively. 
The following Lemma 6 can be easily obtained by using the same statement as Lemma 3.3 in [28].

Lemma 6. ( [28]) Assume that Conditions (C1)-(C3) hold. Let $\left\{z_{n}\right\}$ be a sequence generated by Algorithm 3.2. Then

$$
\begin{aligned}
& \left\|z_{n}-p\right\|^{2} \leq\left\|u_{n}-p\right\|^{2}-\left(1-\mu^{2} \vartheta_{n}^{2} / \vartheta_{n+1}^{2}\right)\left\|u_{n}-y_{n}\right\|^{2}, \quad \forall p \in \operatorname{VI}(\mathcal{C}, A), \\
& \left\|z_{n}-y_{n}\right\| \leq \mu \frac{\vartheta_{n}}{\vartheta_{n+1}}\left\|u_{n}-y_{n}\right\| .
\end{aligned}
$$

Theorem 2. Assume that Conditions (C1)-(C5) hold. Then the sequence $\left\{x_{n}\right\}$ formed by Algorithm 3.2 converges to an element $p \in \operatorname{VI}(\mathcal{C}, A)$ in norm, where $p=P_{\mathrm{VI}(\mathcal{C}, A)}(f(p))$.

Proof. Since $\lim _{n \rightarrow \infty}\left(1-\mu^{2} \vartheta_{n}^{2} / \vartheta_{n+1}^{2}\right)=1-\mu^{2}>0$, there exists $n_{0} \in \mathbb{N}$ such that

$$
1-\mu^{2} \vartheta_{n}^{2} / \vartheta_{n+1}^{2}>0, \quad \forall n \geq n_{0} .
$$

Combining Lemma 6 and (3.15), we get

$$
\left\|z_{n}-p\right\| \leq\left\|u_{n}-p\right\|, \quad \forall n \geq n_{0} .
$$

Claim 1. The sequence $\left\{x_{n}\right\}$ is bounded. Using the same arguments with the Claim 1 in the Theorem 1 , we get that the sequence $\left\{x_{n}\right\}$ is bounded. Consequently, the sequences $\left\{u_{n}\right\},\left\{z_{n}\right\}$ and $\left\{f\left(x_{n}\right)\right\}$ are also bounded.

\section{Claim 2.}

$$
\left(1-\varphi_{n}\right)\left(1-\mu^{2} \vartheta_{n}^{2} / \vartheta_{n+1}^{2}\right)\left\|y_{n}-u_{n}\right\|^{2} \leq\left\|x_{n}-p\right\|^{2}-\left\|x_{n+1}-p\right\|^{2}+\varphi_{n} M_{4} .
$$

Indeed, from Lemma 6 and (3.8), we can immediately get

$$
\begin{aligned}
& \left\|x_{n+1}-p\right\|^{2} \leq \varphi_{n}\left\|x_{n}-p\right\|^{2}+\left(1-\varphi_{n}\right)\left\|z_{n}-p\right\|^{2}+\varphi_{n} M_{3} \\
& \quad \leq\left\|x_{n}-p\right\|^{2}-\left(1-\varphi_{n}\right)\left(1-\mu^{2} \vartheta_{n}^{2} / \vartheta_{n+1}^{2}\right)\left\|y_{n}-u_{n}\right\|^{2}+\varphi_{n} M_{4},
\end{aligned}
$$

where $M_{4}:=M_{2}+M_{3}$, both $M_{2}$ and $M_{3}$ are defined in Claim 2 of Theorem 1 .

\section{Claim 3.}

$$
\begin{aligned}
& \left\|x_{n+1}-p\right\|^{2} \leq\left(1-(1-\kappa) \varphi_{n}\right)\left\|x_{n}-p\right\|^{2}+(1-\kappa) \varphi_{n}\left[\frac{3 M}{1-\kappa} \frac{\varrho_{n}}{\varphi_{n}}\left\|x_{n}-x_{n-1}\right\|\right. \\
& \left.+\frac{2}{1-\kappa}\left\langle f(p)-p, x_{n+1}-p\right\rangle\right], \quad \forall n \geq n_{0} .
\end{aligned}
$$

The desired result can be obtained by using the same arguments as in Claim 3 of Theorem 1.

Claim 4. The sequence $\left\{\left\|x_{n}-p\right\|^{2}\right\}$ converges to zero. According to Claim 4 in Theorem 1, we suppose that $\left\{\left\|x_{n_{k}}-p\right\|\right\}$ is a subsequence of $\left\{\left\|x_{n}-p\right\|\right\}$ satisfying $\liminf \operatorname{int}_{k \rightarrow \infty}\left(\left\|x_{n_{k}+1}-p\right\|-\left\|x_{n_{k}}-p\right\|\right) \geq 0$. From Claim 2 and Condition (C5), one obtains

$$
\limsup _{k \rightarrow \infty}\left(1-\varphi_{n_{k}}\right)\left(1-\mu^{2} \frac{\vartheta_{n_{k}}^{2}}{\vartheta_{n_{k}+1}^{2}}\right)\left\|u_{n_{k}}-y_{n_{k}}\right\|^{2} \leq 0 .
$$


By (3.15), it follows that $\lim _{k \rightarrow \infty}\left\|y_{n_{k}}-u_{n_{k}}\right\|=0$. According to Lemma 6, one has $\lim _{k \rightarrow \infty}\left\|y_{n_{k}}-z_{n_{k}}\right\|=0$. Using the same facts as (3.10)-(3.13), we obtain

$$
\limsup _{k \rightarrow \infty}\left\langle f(p)-p, x_{n_{k}+1}-p\right\rangle \leq 0 .
$$

Therefore, using Claim 3, Condition (C5), (3.16) and Lemma 3, one concludes that $\lim _{n \rightarrow \infty}\left\|x_{n}-p\right\|=0$. The proof is completed.

Remark 3. We have the following observations for the Algorithms 3.1 and 3.2.

(i) Notice that the algorithm proposed by Kraikaew and Saejung [15] is a fixed-step method, i.e., the update of the step size requires the prior information of the Lipschitz constant of the mapping. The algorithms suggested in $[23,30]$ apply an Armijo-type criterion to update the step size, which increases its computational burden by spending a lot of computation in each iteration to find a suitable step size. However, the step size of our two iterative schemes can be updated adaptively without any line search process. In other words, our algorithms do not require the Lipschitz constant as input parameter. In addition, our Algorithms 3.1 and 3.2 embed a new non-monotonic stepsize criterion that overcomes the drawback of non-increasing stepsize sequences generated by the algorithms offered in $[1,2,33]$.

(ii) The algorithms presented in this paper obtain strong convergence theorems in real Hilbert spaces by applying the viscosity-type method. However, the strongly convergent methods presented in $[12,29]$ are obtained by projection-type methods. It is known that projection-based methods are not easy to implement. Therefore, the iterative schemes provided in this paper are more useful.

(iii) The suggested iterative schemes are embedded with inertial effects, which allow them to accelerate the convergence speed of the algorithms $[15,23$, $30,33,34]$ (cf. Section 4).

\section{Numerical examples}

This section reports some numerical results to illustrate the effectiveness of the proposed algorithms in comparisons with some known Algorithms (1.1)(1.5). All the programs were implemented in MATLAB 2018a on a Intel(R) Core(TM) i5-8250U CPU @ 1.60GHz computer with RAM 8.00 GB.

Our parameters are set as follows. For all algorithms, we set $\varphi_{n}=1 /(n+1)$ and $f(x)=0.9 x$. In our proposed algorithms, we choose $\vartheta_{1}=1, \mu=0.9$, $\xi_{n}=1 /(n+1)^{1.1}, \varrho=0.5$ and $\epsilon_{n}=100 /(n+1)^{2}$. Take $\vartheta=0.99 / L$ in Kraikaew and Saejun's Algorithm (1.1). For Shehu and Iyiola's Algorithm (1.2), we set $\rho=0.5$ and $\mu=0.4$. For Thong and Hieu's Algorithm (1.3), we take $\gamma=l=0.5$ and $\mu=0.4$. In Yang et al.'s Algorithm (1.4) and Yang and Liu's Algorithm (1.5), we select $\vartheta_{1}=1$ and $\mu=0.9$. We use $D_{n}=\left\|x_{n}-x^{*}\right\|$ to measure the $n$-th iteration error of all algorithms, where $x^{*}$ represents the solution to our problems. 
Example 1. Consider the linear mapping $A: \mathbb{R}^{m} \rightarrow \mathbb{R}^{m}(m=5,10)$ in the form $A(x)=M x+q$, where $q \in \mathbb{R}^{m}$ and $M=N N^{\top}+U+D, N$ is a $m \times m$ matrix, $U$ is a $m \times m$ skew-symmetric matrix, and $D$ is a $m \times m$ diagonal matrix with its diagonal entries being nonnegative (hence $M$ is positive symmetric definite). The feasible set $\mathcal{C}$ is given by $\mathcal{C}=\left\{x \in \mathbb{R}^{m}:-2 \leq x_{i} \leq 5, i=1, \ldots, m\right\}$. It is clear that $A$ is monotone and Lipschitz continuous with constant $L=\|M\|$. In this experiment, all entries of $N, D$ are generated randomly in $[0,2]$ and $U$ is generated randomly in $[-2,2]$. Let $q=\mathbf{0}$, then the solution set is $x^{*}=$ $\{\mathbf{0}\}$. The maximum number of iterations 400 as the stopping criterion and the initial values $x_{0}=x_{1}$ are randomly generated by $\operatorname{rand}(m, 1)$ in MATLAB. The numerical results are shown in Figure 1.

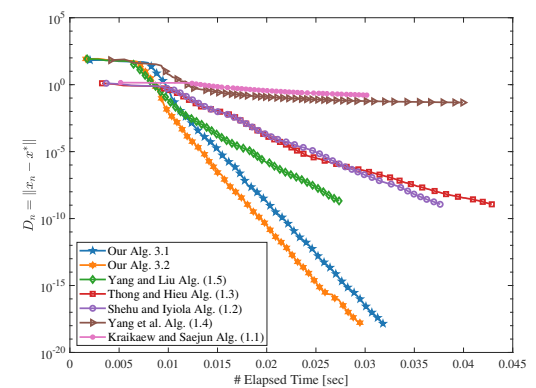

(a) $m=5$

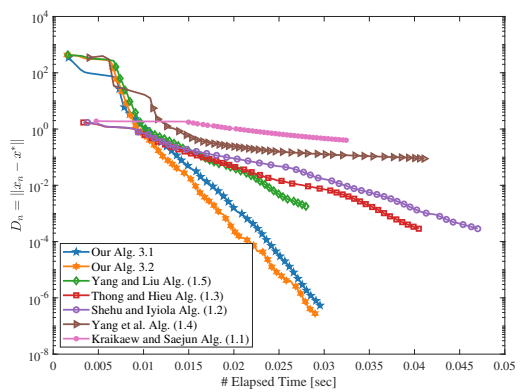

(b) $m=10$

Figure 1. Numerical results for Example 1.

Example 2. In the second numerical experiment, we consider an example in the Hilbert space $\mathcal{H}=L^{2}([0,1])$ associated with the inner product $\langle x, y\rangle:=$ $\int_{0}^{1} x(t) y(t) \mathrm{d} t$ and the induced norm $\|x\|:=\left(\int_{0}^{1}|x(t)|^{2} \mathrm{~d} t\right)^{1 / 2}, \forall x, y \in \mathcal{H}$. Let the feasible set be the unit ball $\mathcal{C}:=\{x \in \mathcal{H}:\|x\| \leq 1\}$. Define a mapping $A: \mathcal{C} \rightarrow \mathcal{H}$ by

$$
(A x)(t)=\int_{0}^{1}(x(t)-G(t, s) g(x(s))) \mathrm{d} s+h(t), \quad t \in[0,1], x \in \mathcal{C},
$$

where

$$
G(t, s)=\frac{2 t s \mathrm{e}^{t+s}}{e \sqrt{\mathrm{e}^{2}-1}}, \quad g(x)=\cos x, \quad h(t)=\frac{2 t \mathrm{e}^{t}}{e \sqrt{\mathrm{e}^{2}-1}} .
$$

It is known that $A$ is monotone and $L$-Lipschitz continuous with $L=2$ and $x^{*}(t)=\{\boldsymbol{0}\}$ is the solution of the corresponding variational inequality problem. Note that the projection on $\mathcal{C}$ is inherently explicit, that is,

$$
P_{\mathcal{C}}(x)= \begin{cases}\frac{x}{\|x\|}, & \text { if }\|x\|>1 \\ x, & \text { if }\|x\| \leq 1\end{cases}
$$

We choose the maximum number of iterations 50 as a common stopping criterion. Figure 2 shows the numerical behaviors of all the algorithms with two starting points. 


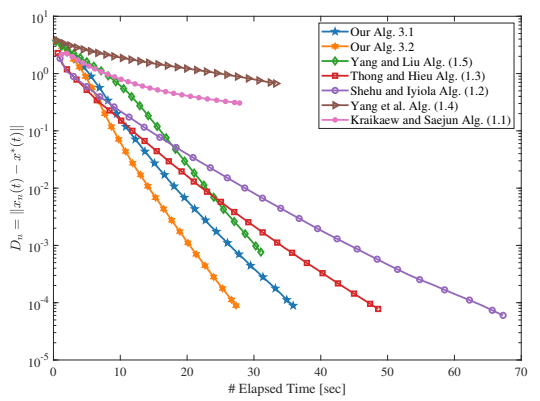

(a) $x_{0}(t)=x_{1}(t)=e^{2 t}$

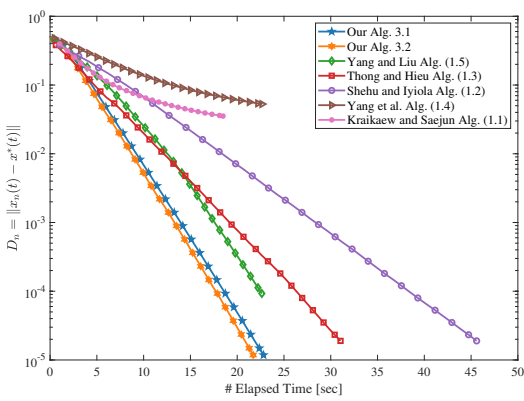

(b) $x_{0}(t)=x_{1}(t)=t^{2}$

Figure 2. Numerical results for Example 2.

Remark 4. From Example 1 and Example 2, we see that our proposed algorithms are outperformance some existing algorithms. The reasonable use of inertial terms and new step size greatly improves the computational performance of our algorithms. Note that the Algorithm (1.2) and the Algorithm (1.3) require more execution time because they use an Armijo-like line search method to adaptively calculate the step size.

Next, we use the proposed Algorithms 3.1 and 3.2 to solve variational inequalities that appears in optimal control problems. Recently, many scholars have proposed different methods to solve it. We recommend readers to refer to $[8,19,32]$ for the algorithms and detailed description of the problem.

Example 3. (Control of a harmonic oscillator, see [18])

$$
\begin{aligned}
\operatorname{minimize} & x_{2}(3 \pi) \\
\text { subject to } & \dot{x}_{1}(t)=x_{2}(t), \\
& \dot{x}_{2}(t)=-x_{1}(t)+u(t), \quad \forall t \in[0,3 \pi], \\
& x(0)=0, \quad u(t) \in[-1,1] .
\end{aligned}
$$

The exact optimal control of Example 3 is known:

$$
u^{*}(t)=\left\{\begin{aligned}
1, & \text { if } t \in[0, \pi / 2) \cup(3 \pi / 2,5 \pi / 2), \\
-1, & \text { if } t \in(\pi / 2,3 \pi / 2) \cup(5 \pi / 2,3 \pi]
\end{aligned}\right.
$$

In the suggested Algorithms 3.1 and 3.2, we set $N=100, \mu=0.1, \vartheta_{1}=0.4$, $\xi_{n}=1 /(n+1)^{1.1}, \varrho=0.3, \epsilon_{n}=10^{-4} /(n+1)^{2}, \varphi_{n}=10^{-4} /(n+1)$ and $f(x)=$ $0.1 x$. The initial controls $u_{0}(t)=u_{1}(t)$ are randomly generated in $[-1,1]$, and the stopping criterion is $\left\|u_{n+1}-u_{n}\right\| \leq 10^{-4}$ or maximum iteration 1000 times. Algorithm 3.1 and Algorithm 3.2 take 0.14205 seconds and 0.045983 seconds to reach the allowable error, respectively. Figure 3 shows the approximate optimal control and the corresponding trajectories of Algorithm 3.1.

We now consider an example in which the terminal function is not linear. 


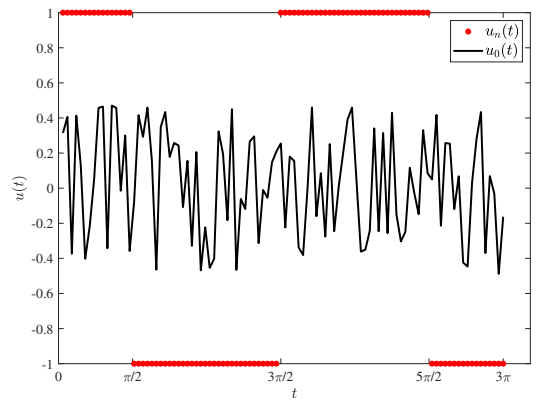

(a) Initial and optimal controls

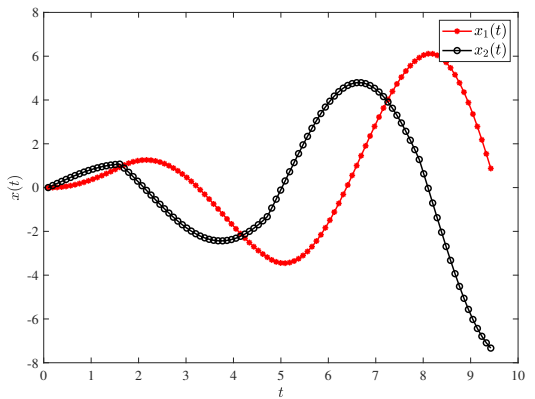

(b) Optimal trajectories

Figure 3. Numerical results for Example 3.

Example 4. (Rocket car [19])

$$
\begin{array}{cl}
\operatorname{minimize} & \frac{1}{2}\left(\left(x_{1}(5)\right)^{2}+\left(x_{2}(5)\right)^{2}\right) \\
\text { subject to } & \dot{x}_{1}(t)=x_{2}(t), \quad \dot{x}_{2}(t)=u(t), \quad \forall t \in[0,5], \\
& x_{1}(0)=6, \quad x_{2}(0)=1, \quad u(t) \in[-1,1] .
\end{array}
$$

The exact optimal control of Example 4 is

$$
u^{*}(t)=\left\{\begin{aligned}
1, & \text { if } t \in(3.517,5] \\
-1, & \text { if } t \in(0,3.517]
\end{aligned}\right.
$$

In this example, the parameters of our algorithms are set the same as in Example 3. Algorithm 3.1 ran 644 iterations and Algorithm 3.2 ran 1000 iterations, which take 0.28734 seconds and 0.39556 seconds, respectively. The approximate optimal control and the corresponding trajectories of Algorithm 3.2 are plotted in Figure 4.

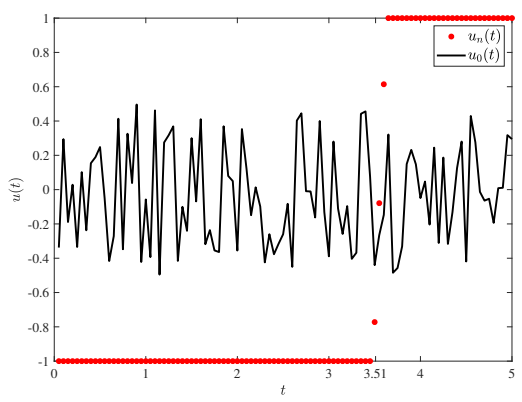

(a) Initial and optimal controls

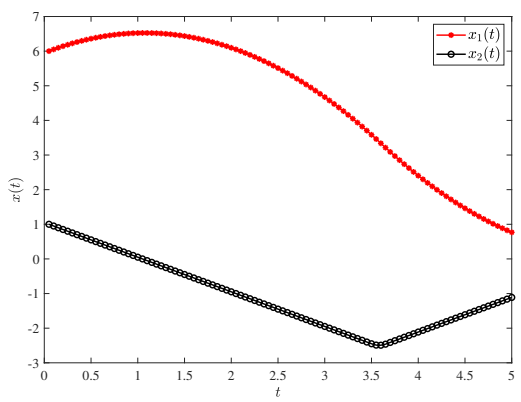

(b) Optimal trajectories

Figure 4. Numerical results for Example 4. 
Remark 5. As can be seen from Examples 3 and 4, the algorithms proposed in this paper can work well on optimal control problems. It is worth noting that when the objective function is linear rather than nonlinear, our suggested algorithms can work better (cf. Figures 3 and 4).

\section{Conclusions}

In this paper, we proposed two new algorithms for solving the variational inequality problem with a monotone and Lipschitz continuous mapping but the Lipschitz constant is unknown. The algorithms are inspired by the inertial method, the subgradient extragradient method, the Tseng's extragradient method and the viscosity method. Strong convergence theorems of the proposed algorithms were obtained under some mild and standard conditions. Finally, some numerical experiments arise in finite- and infinite-dimensional spaces are performed to show the efficiency and advantages of our suggested iterative schemes over the existing ones.

\section{Acknowledgements}

The authors would like to thank the editors and the referees for their valuable comments and suggestions, which greatly improved the readability and quality of the paper.

\section{References}

[1] T.O. Alakoya, L.O. Jolaoso and O.T. Mewomo. Two modifications of the inertial tseng extragradient method with self-adaptive step size for solving monotone variational inequality problems. Demonstratio Mathematica, 53(1):208-224, 2020. https://doi.org/10.1515/dema-2020-0013.

[2] T.O. Alakoya, L.O. Jolaoso and O.T. Mewomo. Modified inertial subgradient extragradient method with self adaptive stepsize for solving monotone variational inequality and fixed point problems. Optimization, 70(3):545-574, 2021. https://doi.org/10.1080/02331934.2020.1723586.

[3] T.O. Alakoya, A. Taiwo, O.T. Mewomo and Y.J. Cho. An iterative algorithm for solving variational inequality, generalized mixed equilibrium, convex minimization and zeros problems for a class of nonexpansive-type mappings. Ann Univ Ferrara, 67:1-31, 2021. https://doi.org/10.1007/s11565-020-00354-2.

[4] Q.H. Ansari, M. Islam and J.-Ch. Yao. Nonsmooth variational inequalities on Hadamard manifolds. Applicable Analysis, 99(2):340-358, 2020. https://doi.org/10.1080/00036811.2018.1495329.

[5] Y. Censor, A. Gibali and S. Reich. Strong convergence of subgradient extragradient methods for the variational inequality problem in Hilbert space. Optimization Methods and Software, 26(4-5):827-845, 2011. https://doi.org/10.1080/10556788.2010.551536.

[6] T.H. Cuong, J.-Ch. Yao and N.D. Yen. Qualitative properties of the minimum sum-of-squares clustering problem. Optimization, 69(9):2131-2154, 2020. https://doi.org/10.1080/02331934.2020.1778685. 
[7] A. Gibali and D.V. Hieu. A new inertial double-projection method for solving variational inequalities. Journal of Fixed Point Theory and Applications, 21(4), 2019. https://doi.org/10.1007/s11784-019-0726-7.

[8] D.V. Hieu, J.J. Strodiot and L.D. Muu. Strongly convergent algorithms by using new adaptive regularization parameter for equilibrium problems. Journal of Computational and Applied Mathematics, 376, 2020. https://doi.org/10.1016/j.cam.2020.112844.

[9] C. Izuchukwu, A.A. Mebawondu and O.T. Mewomo. A new method for solving split variational inequality problems without co-coerciveness. Journal of Fixed Point Theory and Applications, 22(98), 2020. https://doi.org/10.1007/s11784020-00834-0.

[10] C. Izuchukwu, G.N. Ogwo and O.T. Mewomo. An inertial method for solving generalized split feasibility problems over the solution set of monotone variational inclusions. Optimization, pp. 1-29, 2020. https://doi.org/10.1080/02331934.2020.1808648.

[11] L.O. Jolaoso, T.O. Alakoya, A. Taiwo and O.T. Mewomo. Inertial extragradient method via viscosity approximation approach for solving equilibrium problem in Hilbert space. Optimization, 70(2):387-412, 2021. https://doi.org/10.1080/02331934.2020.1716752.

[12] L.O. Jolaoso, A. Taiwo, T.O. Alakoya and O.T. Mewomo. A strong convergence theorem for solving pseudo-monotone variational inequalities using projection methods. Journal of Optimization Theory and Applications, 185:744-766, 2020. https://doi.org/10.1007/s10957-020-01672-3.

[13] A.A. Khan, S. Migorski and M. Sama. Inverse problems for multivalued quasi variational inequalities and noncoercive variational inequalities with noisy data. Optimization, 68(10):1897-1931, 2019. https://doi.org/10.1080/02331934.2019.1604706.

[14] G.M. Korpelevich. The extragradient method for finding saddle points and other problems. Ekonomika i Matematicheskie Metody, 12(4):747-756, 1976.

[15] R. Kraikaew and S. Saejung. Strong convergence of the halpern subgradient extragradient method for solving variational inequalities in Hilbert spaces. Journal of Optimization Theory and Applications, 163:399-412, 2014. https://doi.org/10.1007/s10957-013-0494-2.

[16] H. Liu and J. Yang. Weak convergence of iterative methods for solving quasimonotone variational inequalities. Computational Optimization and Applications, 77:491-508, 2020. https://doi.org/10.1007/s10589-020-00217-8.

[17] L. Liu. A hybrid steepest descent method for solving split feasibility problems involving nonexpansive mappings. Journal of Nonlinear and Convex Analysis, 20(3):471-488, 2019.

[18] A. Pietrus, T. Scarinci and V.M. Veliov. High order discrete approximations to Mayer's problems for linear systems. SIAM Journal on Control and Optimization, 56(1):102-119, 2018. https://doi.org/10.1137/16M1079142.

[19] J. Preininger and P.T. Vuong. On the convergence of the gradient projection method for convex optimal control problems with bang-bang solutions. Computational Optimization and Applications, 70:221-238, 2018. https://doi.org/10.1007/s10589-018-9981-6. 
[20] S. Saejung and P. Yotkaew. Approximation of zeros of inverse strongly monotone mappings in Banach spaces. Nonlinear Analysis: Theory, Methods \& Applications, 75(2):742-750, 2012. https://doi.org/10.1016/j.na.2011.09.005.

[21] D.R. Sahu, J.C. Yao, M. Verma and K.K. Shukla. Convergence rate analysis of proximal gradient methods with applications to composite minimization problems. Optimization, $\mathbf{7 0}(1): 75-100,2021$. https://doi.org/10.1080/02331934.2019.1702040.

[22] Y. Shehu and A. Gibali. New inertial relaxed method for solving split feasibilities. Optimization Letters, 15:2109-2126, 2021. https://doi.org/10.1007/s11590-02001603-1.

[23] Y. Shehu and O. Iyiola. Strong convergence result for monotone variational inequalities. Numerical Algorithms, 76:259-282, 2017. https://doi.org/10.1007/s11075-016-0253-1.

[24] A. Taiwo, T.O. Alakoya and O.T. Mewomo. Halpern-type iterative process for solving split common fixed point and monotone variational inclusion problem between Banach spaces. Numerical Algorithms, 86:1359-1389, 2021. https://doi.org/10.1007/s11075-020-00937-2.

[25] A. Taiwo, L.O. Jolaoso and O.T. Mewomo. Inertial-type algorithm for solving split common fixed-point problem in Banach spaces. Journal of Scientific Computing, 86, 2021. https://doi.org/10.1007/s10915-020-01385-9.

[26] W. Takahashi. Nonlinear Functional Analysis. Yokohama Publishers, Yokohama, 2000 .

[27] W. Takahashi, C.F. Wen and J.-Ch. Yao. The shrinking projection method for a finite family of demimetric mappings with variational inequality problems in a Hilbert space. Fixed Point Theory, 19(1):407-420, 2018. https://doi.org/10.24193/fpt-ro.2018.1.32.

[28] B. Tan, J. Fan and S. Li. Self-adaptive inertial extragradient algorithms for solving variational inequality problems. Computational and Applied Mathematics, 40(19), 2021. https://doi.org/10.1007/s40314-020-01393-3.

[29] B. Tan, S. Xu and S. Li. Inertial shrinking projection algorithms for solving hierarchical variational inequality problems. Journal of Nonlinear and Convex Analysis, 21(4):871-884, 2020.

[30] D.V. Thong and D.V. Hieu. Weak and strong convergence theorems for variational inequality problems. Numerical Algorithms, 78:1045-1060, 2018. https://doi.org/10.1007/s11075-017-0412-z.

[31] P. Tseng. A modified forward-backward splitting method for maximal monotone mappings. SIAM Journal on Control and Optimization, 38(2):431-446, 2000. https://doi.org/10.1137/S0363012998338806.

[32] P.T. Vuong and Y. Shehu. Convergence of an extragradient-type method for variational inequality with applications to optimal control problems. Numerical Algorithms, 81:269-291, 2019. https://doi.org/10.1007/s11075-018-0547-6.

[33] J. Yang and H. Liu. Strong convergence result for solving monotone variational inequalities in Hilbert space. Numerical Algorithms, 80:741-752, 2019. https://doi.org/10.1007/s11075-018-0504-4.

[34] J. Yang, H. Liu and Z. Liu. Modified subgradient extragradient algorithms for solving monotone variational inequalities. Optimization, 67(12):2247-2258, 2018. https://doi.org/10.1080/02331934.2018.1523404. 